\title{
Correlation in narrow nanorods: a variational potential-configuration interaction scheme
}

\author{
J. Planelles, J.I. Climente, M. Royo, and J.L. Movilla \\ Departament de Química-Física i Analítica, UJI, Box 224, E-12080 Castelló, Spain \\ E-mail: josep.planelles@qfa.uji.es
}

\begin{abstract}
Full configuration interaction calculations of two electrons in narrow semiconductor nanorods are carried out employing different orbital basis sets. It is shown that the usual configurations built from single-particle states cannot yield a correct singlet-triplet energetic order regardless of the basis size, as they miss correlation energy. Mean-field optimized orbitals partially correct this drawback. A new approach is introduced, based on a simple variational procedure, which yields robust results.
\end{abstract}

PACS numbers: 71.15.-m, 71.27.+a, 73.21.La 


\section{Introduction}

Quantum dots (QDs) are artificial semiconductor structures with sizes a few of tens nanometers. Often, they are modeled as local perturbations of the periodic crystal field of semiconductor surrounding matrix, using envelope function and effective mass approximations to describe their electronic structure.[1, 2] Within this approach the details of the unit cell are integrated out, so that only a macroscopic (or envelope) description of the system remains. Thus, the interaction between conduction electrons and atomic core electrons and nuclei is averaged as the interaction with a continuous polarizable medium. Likewise, Coulomb interaction between carriers is assumed to be screened by the dielectric constant of the QD bulk material.[3] The large value of the semiconductor dielectric constant coming into the Coulomb term (typically 10-14), along with the small value of the electron effective mass coming into the kinetic energy term, often lead to situations in which the separation between discrete single-particle (SP) levels exceeds by far the characteristic interaction energy between particles. As a consequence, SP descriptions of the many-body problem may be used, treating the Coulomb interaction between conduction electrons as a perturbation. $[4,5,6]$

A better accounting for the two-particle interaction can be achieved through diagonalization of the many-body Hamiltonian in the basis set of non-interacting configurations. It is the so-called full configuration interaction (FCI) method, which plays a central role in quantum chemistry.[7] FCI provides benchmark results for the ground state energy and wave function, as well as for the description of excited states, as it is inferred from the McDonald theorem.[8] The drawback of the approach is its computational cost, originated from its often slow convergence. The key for convergence is the selection of a suitable monoelectronic basis set. As showed by Löwdin and Shull, [9] the basis of natural orbitals is the one which requires the fewest configurations to achieve a given accuracy in the energy. Natural orbitals are the orbitals that diagonalize the one-particle density matrix. Therefore, exact natural orbitals should be extracted from the FCI wave function. Alternatively, one may use single particle (SP) orbitals, i.e., the eigenfunctions of the one-body operator coming into the many-body Hamiltonian. This approach is quite common in few-electron and excitonic QD calculations, where convergence problems do not usually arise. $[4,5,10]$

In this paper, however, we identify a QD system where correlation energies are so strong that the basis choice becomes critical to overcome slow convergence issues. Namely, we study narrow rod-shaped QDs (nanorods, NRs).[11, 12] As recently shown, the large aspect ratio of these semiconductor structures is responsible for remarkably strong Coulomb interactions. [13, 14, 15] Here we demonstrate that, even in the simplest case of two interacting electrons, the CI method based on SP orbitals (SP-CI) fails to converge. Indeed, for long rods it predicts a triplet ground state, thus violating a fundamental theorem for two-body exact functions.[16] To solve this problem, we follow quantum chemistry CI methods and replace the regular SP orbitals by effective 
orbitals obtained through mean field calculations, either Hartree-Fock (HF-CI)[7] or Kohn-Sham density functional theory (KS-CI). [17] The suitability of using these meanfield optimized orbitals is discussed and compared with a new, simpler approach based on a two-fold variational procedure.

\section{Theory}

The Hamiltonian of interacting conduction electrons in a QD reads

$$
\mathcal{H}=\sum_{i} T(i)+v^{c}(i)+\sum_{j<i} \frac{1}{\epsilon r_{i j}}=\sum_{i} h(i)+\sum_{j<i} g(i, j),
$$

where $T=-\frac{1}{2 m^{*}} \nabla^{2}$ is the kinetic energy operator, $v^{c}$ the spatial confining potential, $m^{*}$ the effective mass and $\epsilon$ the dielectric constant.

In order to carry out a CI calculation, first one has to select a given oneelectron basis set $\left\{\phi_{p}\right\}_{p=1}^{M}$ and then build all possible N-body Salter determinants $\Phi_{\alpha}=\operatorname{det}\left\{\phi_{1} \phi_{2} \ldots \phi_{N}\right\}$ out of them. In a second step, the projection of Hamiltonian (1) onto the basis set of Slater determinants (or onto a subset of spin- and symmetryadapted configurations) is carried out yielding matrix elements $\left\langle\Phi_{\alpha}|\mathcal{H}| \Phi_{\beta}\right\rangle$. The last step is the diagonalization.

For practical purposes the one-electron basis set is taken orthogonal, although many-body approaches based on overlapping orbitals, as e.g. the generalized valence bond (GVB), have been successfully developed in quantum chemistry.[7, 18] A possible orbital choice is the SP states, i.e., the eigenfunctions of one-electron Hamiltonian $h$. We must select a finite basis set. The natural choice is the $M$ lowest-energy eigenvectors. This truncation of the basis set implies a projection of Hamiltonian (1) onto the corresponding FCI space yielding a model Hamiltonian that, in second quantization language, reads,

$$
H_{S P}=\sum_{p}^{M} \varepsilon_{p} a_{p}^{+} a_{p}+\sum_{p q r s}^{M} g_{p q r s} a_{p}^{+} a_{q}^{+} a_{r} a_{s}
$$

where $\varepsilon_{p}=h_{p p}$ is the p-th eigenvalue of $h, g_{p q r s}$ the two-electron integral and $a_{p}^{+}, a_{q}$ the usual fermion creator/annihilator operators.

We may, alternatively, employ another M-dimensional orthogonal basis set $\left\{\chi_{p}\right\}_{p=1}^{M}$. In this case, Hamiltonian (1) turns into,

$$
H_{X}=\sum_{p q}^{M} h_{p q} a_{p}^{+} a_{q}+\sum_{p q r s}^{M} g_{p q r s} a_{p}^{+} a_{q}^{+} a_{r} a_{s}
$$

where off-diagonal $h_{p q}$ terms arise because $\chi_{p}$ is no longer an eigenfunction of $h$. 
We may consider $\left\{\chi_{p}\right\}$ as related to a given $v^{m f}(r)$ potential operator which is added and subtracted to Hamiltonian (1),

$$
\begin{aligned}
\mathcal{H} & =\sum_{i} h(i)+v^{m f}(i)-v^{m f}(i)+\sum_{j<i} g(i, j) \\
& =\sum_{i}\left(h(i)+v^{m f}(i)+\sum_{j<i} g(i, j)-v^{m f}(i)\right) \\
& =\sum_{i} f(i)+\sum_{j<i} \bar{g}(i, j) .
\end{aligned}
$$

Now we determine $\left\{\chi_{p}\right\}$ from the eigenvalue equation $f(r) \chi_{p}(r)=\bar{\varepsilon}_{p} \chi_{p}(r)$ and rewrite eq. (3) as

$$
H_{X}=\sum_{p}^{M} \bar{\varepsilon}_{p} a_{p}^{+} a_{p}+\sum_{p q r s}^{M} \bar{g}_{p q r s} a_{p}^{+} a_{q}^{+} a_{r} a_{s},
$$

HF, density functional theory (DFT) and, in general, any mean-field calculation are particular cases of this procedure. For example, in HF,

$$
\bar{\varepsilon}_{p}=h_{p p}+\sum_{q}^{M}\left(\left\langle p q\left|\frac{1}{\epsilon r_{12}}\right| p q\right\rangle-\left\langle p q\left|\frac{1}{\epsilon r_{12}}\right| q p\right\rangle\right)
$$

Note that $h_{p q}+v_{p q}^{m f}=\left\langle p\left|h+v^{m f}\right| q\right\rangle=\bar{\varepsilon}_{p}\langle p \mid q\rangle=\bar{\varepsilon}_{p} \delta_{p q}$.

Within the framework of this procedure, our approach to deal with strongly correlated regimes is the use of a variational potential, i.e., a potential depending on parameters that allow optimization of the orbital basis set in the CI process.

The advantadge of adding a mean field potential is that, contrary to the SP orbitals which disregard electron-electron interaction, HF orbitals include it somehow averaged in the Coulomb and exchange terms, and KS orbitals further account for some of the correlation through the corresponding functional. This has proved useful in describing many-electron QDs.[19, 20] In our approach, all terms in Hamiltonian (1) are treated on equal footing to optimize the potential and hence the orbital basis set.

It is worth noting that the projected Hamiltonian (3) is not defined by the selected orbital basis set but rather by the linear space that it generates. Thus for example, in [19], where HF spinorbitals of a $N$-electron system are expressed as linear combination of a set of $K$ SP states, a FCI expansion which employs either $K$-dimensional basis set yields identical result. The advantage of the strategy presented in [19] relies on a further truncation of the HF basis set. 


\section{Results and discussion}

We carry out calculations of the lowest-lying singlet and triplet states of a two-electron semiconductor CdSe NR. The rod is composed by a cylinder of radius $R=20 \AA$ and variable length $L$, attached to two hemispherical caps of the same radius $R$ in the extremes. The material parameters are effective mass $m^{*}=0.13$, dielectric constant $\epsilon=9.2$ and confining potential $v^{c}=4 \mathrm{eV}$. The dominant electronic configuration for the studied singlet and triplet states are $\sigma_{1}^{2}$ and $\sigma_{1} \sigma_{2}$, respectively, where $\sigma_{1}, \sigma_{2}$ are the lowest-lying and first excited orbitals with zero $z$-component of the angular momentum $\left(M_{z}=0\right)$. We have checked that, as expected, already in the case of a short NR, many $\sigma\left(M_{z}=0\right)$ SP states lie energetically below the first $\pi\left(M_{z}= \pm 1\right)$ energy level, and that higher angular momentum states are much more excited. Then, we employ a basis set $1-n-1$ in the CI calculations, i.e., a basis set including the $n$ lowest-lying $\sigma$ and the lowest-lying degenerate pair of $\pi$ orbitals, the last orbital pair having, in all studied cases, a very small contribution to the singlet and triplet CI wave functions.

Figure 1 shows a series of SP-CI calculations (basis set 1-6-1) of the singlet and triplet states of a $R=20 \AA$ two-electron semiconductor CdSe NR vs. L. At first glance, the results seem reasonable. At $L=0$ (sphere) the singlet state lies energetically far below the triplet state, and the contributions to the CI expansion of configurations others than the dominant one are extremely small. As $L$ increases, the energetic singlet-triplet gap $\Delta_{T S}$ decreases, the contribution of excited configurations to the CI wave function increases and, in the large $L$ limit a singlet-triplet degeneration occurs. However, a closer view reveals that beyond $L=170 \AA$ the triplet state becomes the ground state (see inset in Fig.1). This fact is a violation of a known theorem, attributed to Wigner, stating that any general two-particle Hamiltonian including a symmetric and real potential energy operator has a singlet ground state.[16] Therefore, the result is qualitatively wrong. One may relate the failure to the truncation of the orbital basis set. In order to check it, we run a series of SP-CI calculations of the singlet-triplet energy gap $\Delta_{T S}$ in an $L=200 \AA \mathrm{NR}$ vs. the size $n$ of the (1-n-1) basis set. The results, shown in Figure 2 , do not allow to foresee any finite $n$ yielding the theoretically expected singlet ground state.

We turn then our attention to the wave function. The inset in Figure 2 shows a profile of the FCI singlet (solid line) and triplet (dashed line) charge densities along the $\mathrm{NR}$ vertical axis. We can observe that the triplet and, to a lesser extent, the singlet state develop a valley in the electronic density profile around the rod center. The presence of this valley allows the interacting electrons to reduce the repulsion energy, hence stabilizing the states. This groove is already present in the independent-particle description of the triplet state (through the $\sigma_{2}$ orbital), but not in the singlet ground state, where it is a pure correlation effect.[15] In the case of a poor correlation description, the singlet valley is not deep enough and the triplet is more stable, leading to the triplet ground 
state predicted above.

Since the singlet state has gerade symmetry and only double excitations to ungerade orbitals may contribute to the formation of the central valley in the density profile of the singlet, we explore the possibility of carrying out a CI expansion using gerade orbitals with an in-built valley. A first attempt is to follow a recent approach proposed by Abolfath and Hawrylak of using HF orbitals.[19] However, the approach is useless in our case, because in this scheme HF orbitals are expanded in terms of a (large) SP basis set and, as shown in Fig. 2, even an extremely large (finite) SP basis set cannot prevent the singlet-triplet reversal. Note that gerade/ungerade HF orbitals would be linear combinations of gerade/ungerade SP orbitals. Therefore, no gerade HF orbital developing a valley can be obtained as linear combination of SP orbitals. We then take a different approach and carry out numerical HF calculations, build a many-electron Hamiltonian (5) out of the $M$ lowest-lying HF eigenvectors and repeat the calculations shown in Figure 1 with this new orbital basis set. In particular, the results collected in Figure 3 corresponds to a 1-6-1 HF basis set. One can see that HF-CI outperforms SP-CI. Indeed, at $L=170 \AA$, HF-CI clearly predicts a singlet ground state. All the same, a singlet-triplet crossing is observed shortly after $L=200 \AA$, indicating that the basis set is still insufficient.

We investigate with some more detail the pathological region of large $L$. To this end, we consider an $L=200 \AA \mathrm{NR}$, and carry out calculations employing a large basis set (1-10-1). In a first instance we run mean-field HF and KS (in the local density approximation) calculations. The corresponding mean field potentials are represented in Figure 4 left and center, respectively. We see that both have similar shape, i.e. a profile vs. $z$ resembling a symmetric hill with the top at the rod center. In Figure 4 (right) we show a simplified potential $v^{t r i}$, composed by a triangle of height $H$ along the NR $z$-axis. All three potentials have a common feature: they unstabilize the central region of the NR. In the case of $\mathrm{HF}$ and $\mathrm{KS}$, the mean-field potentials are optimized in a self-consistent procedure. As far as the third potential is concerned, we optimized the height $H$ of $v^{t r i}$ variationally, i.e., we perform FCI calculations with states obtained as eigenvectors of a modified SP Hamiltonian, which adds $v^{\text {tri }}(H)$ to the confining potential, and then select variationally the best $H$. This optimization is shown in Figure 5 for a $n=10$ basis set. We represent singlet and triplet FCI energies vs. the triangle height $H$. For comparison, horizontal lines representing HF-FCI and KS-FCI are enclosed. Several relevant conclusions that may be drawn from this figure are: (i) all three potentials recover the correct singlet/triplet energetic ordering; (ii) orbital optimization affects the singlet state far more than the triplet; (iii) from the variational principle and Mcdonald's theorem, we conclude that $v^{K S}$ outperforms $v^{H F}$ and the variational potential $v^{t r i}$ outperforms both, $v^{H F}$ and $v^{K S}$. The good performance of $v^{K S}$ is remarkable in view of its typical failures in very-few electron systems[21]. Also, we note that in this system HF-CI gives better results than the SP-CI scheme, contrary to the 
expectations of [19] for a low number of electrons. The singlet-triplet $\Delta_{T S}$ gap yielded by $v^{H F}, v^{K S}$ and $v^{t r i}$ through the FCI calculations are $0.2,1.4$ and $3.5 \mathrm{meV}$, respectively.

In a next step, we consider again the 1-6-1 basis set employed in Figures 1 and 3 , run calculations optimizing $v^{\text {tri }}$ in the region $100 \AA \leq \mathrm{L} \leq 250 \AA$, and compare the results yielded by $v^{t r i}$ with the SP-CI, and HF-CI methods. The combined results are summarized in Figure 6, where the FCI singlet-triplet gap $\Delta_{T S}$ obtained from SP, $v^{H F}$ and $v^{t r i}$ vs. $L$, is represented. One can see that only in the case of $v^{t r i}$ the correct null asymptotic limit of the singlet-triplet gap is obtained. In the inset, the difference between the calculated SP or HF gaps and the benchmark one $\left(v^{\text {tri }}\right)$ is displayed. The inset shows that, within this region, the gap error is approximately constant for both approaches. It mainly comes from a deficient description of the singlet state (see Figure 5). An analysis of the orbitals employed in the different calculations reveals that an optimal description of the singlet state with a moderate-size basis set requires a well developed valley around the rod center in the ground $\sigma_{g}$ orbital, but the valley should be smaller o even be absent in excited $\sigma_{g}$ orbitals.

To close this section we would like to note that the poor correlation description of SP-CI is exclusive of NRs with large aspect ratio. As a matter of fact, SP-CI calculations for a $R=50 \AA, L=200 \AA$ (total length $300 \AA$ ) CdSe NR employing a 1-6-1 basis set, yields the correct singlet-triplet order, contrary to the $R=20 \AA \mathrm{NR}$ we have analyzed in detail.

\section{Conclusion}

We have shown that the FCI correlation energy of two electrons in quasi-one-dimensional NRs calculated with a usual orbital basis set of numerical eigenfunctions of the oneelectron operator $h$ arising in the many-body Hamiltonian (SP-FCI scheme) leads to a wrong singlet-triplet energetic order. Irrespective of basis size, the correlation energy of the NR is very sensitive to the choice of orbitals. Thus, mean-field-CI approaches, that take into account somehow averaged the electron-electron interaction in the orbital optimization can partially correct this drawback. A new CI scheme is proposed in which the mean field potential is replaced by a simple triangular potential, with the triangle height as a variational parameter. The latter approach outperforms mean-fieldCI schemes, yielding robuts results.

\section{Acknowledgments}

Financial support from MCI project CTQ2008-03344, UJI-Bancaixa project P1-1B200603, the Ramon y Cajal program (JIC) and a Generalitat Valenciana FPI grant (MR) is 
Correlation in narrow nanorods: a variational potential-configuration interaction scheme8

gratefully acknowledged.

[1] Bastard G, 1988 Wave Mechanics Applied to Semiconductor Heterostructures, (Les Éditions de Physique, Les Ulis).

[2] Singh J, 2003 Electronic and Optoelectronic Propoerties of Semiconductor Structures, (Cambridge University Press, Cambridge).

[3] Bányai L and Koch SW, 1993 Semiconductor Quantum Dots (Word Scientific, Singapore).

[4] Jacak L, Hawrylak P and Wós A, 1998 Quantum Dots (Springer, Berlin).

[5] Chakraborty T, 1999 Quantum Dots,(Elsevier Science, Amsterdam).

[6] Warburton RJ, Miller MT, Dürr CS, Bödefeld C, Karrai K, Kotthaus JP, Medeiros-Ribeiro G, Petroff PM, and Huant S, Phys. Rev. B 58, 16221 (1998).

[7] Szabo A and Ostlund NS, 1989 Modern Quantum Chemistry, (McGraw-Hill, New York).

[8] McDonald JKL, Phys. Rev. 43, 830 (1933).

[9] Löwdin PO and Shull H, Rev. Mod. Phys. 101, 1730 (1956).

[10] Wensauer A, Korkusinski M and Hawrylak P, Sol. Stat. Commun. 130, 115 (2004).

[11] Peng X, Manna L, Yang W, Wickham J, Scher E, Kadavanich A, and Alivisatos AP, Nature 404, $59(2000)$.

[12] Kan S, Mokari T, Rothenberg E, and Banin U, Nature Materials 2, 155 (2003).

[13] Shabaev A, and Efros Al.L., Nano Lett. 4, 1821 (2004).

[14] Baskoutas S, Chem. Phys. Lett. 404, 107 (2005).

[15] Climente JI, Royo M, Movilla JL, and Planelles J, submitted.

[16] Lieb E and Mattis D, Phys. Rev. 125, 164 (1962).

[17] Bour P, Chem. Phys. Lett. 345, 331 (2001); Grimme S and Waletzke M, J. Chem. Phys. 111, 5645 (1999)

[18] Goddard WA, Dunning TH, Hunt WJ and Hay PJ, Acc. Chem. Res. 6, 368 (1973).

[19] Abolfath RM and Hawrylak P, J. Chem. Phys. 125, 034707 (2006)

[20] Emperador A, Lipparini E and Pederiva F, Phys. Rev. B 72, 033306 (2005).

[21] Legrand C, Suraud E and Reinhard PG, J. Phys. B: At. Mol. Opt. Phys. 35, 1115 (2002); Chwiej T, Bednarek S, Szafran C, Adamowski J and Peeters FM, Phys. Rev. B 73, 075422 (2006). 


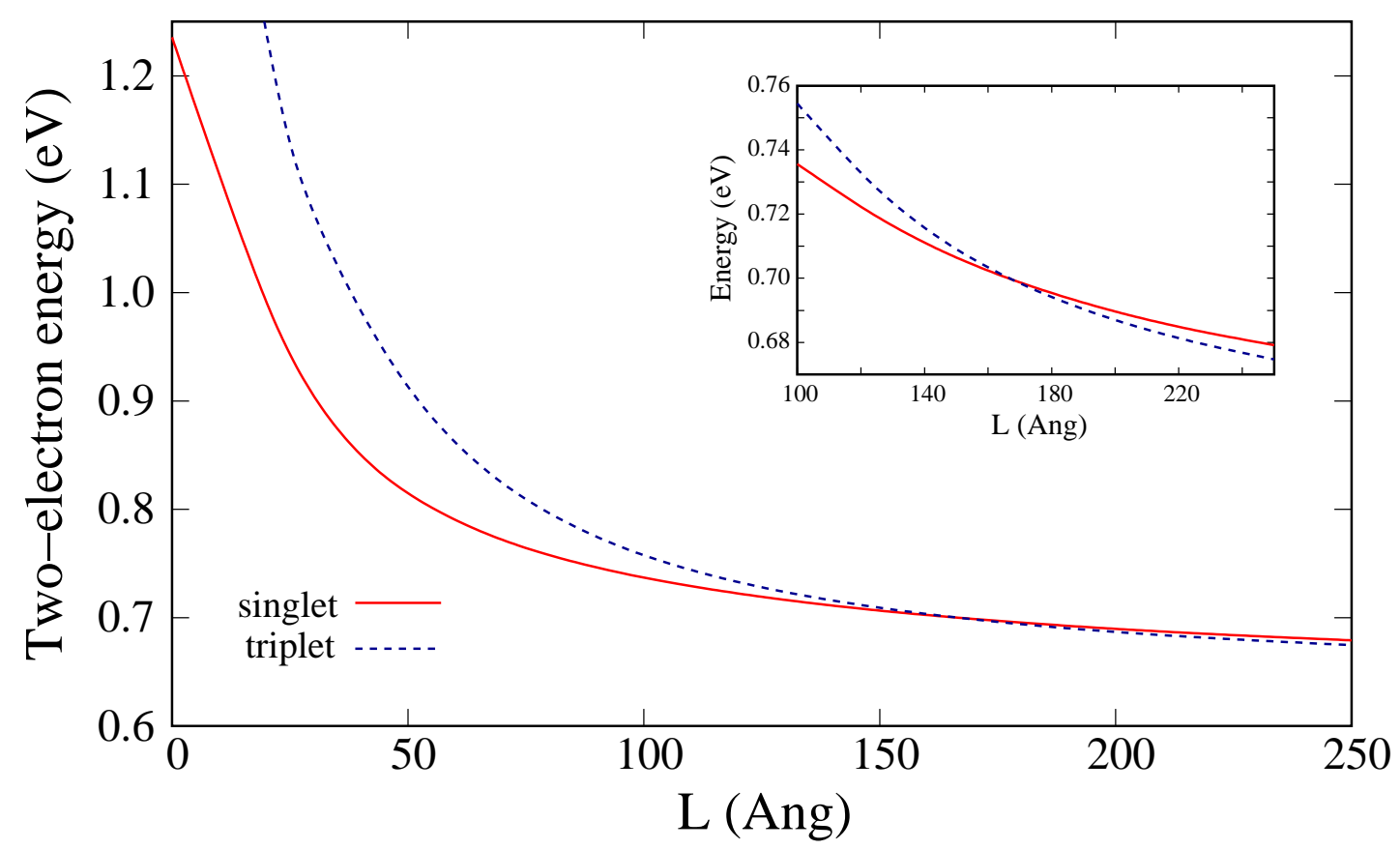

Figure 1. FCI calculations, employing a 1-6-1 SP basis set, of the singlet (solid line) and triplet (dashed line) states of a $R=20 \AA$ two-electron semiconductor CdSe NR vs. the length $L$. Inset: close-up of the large length region.

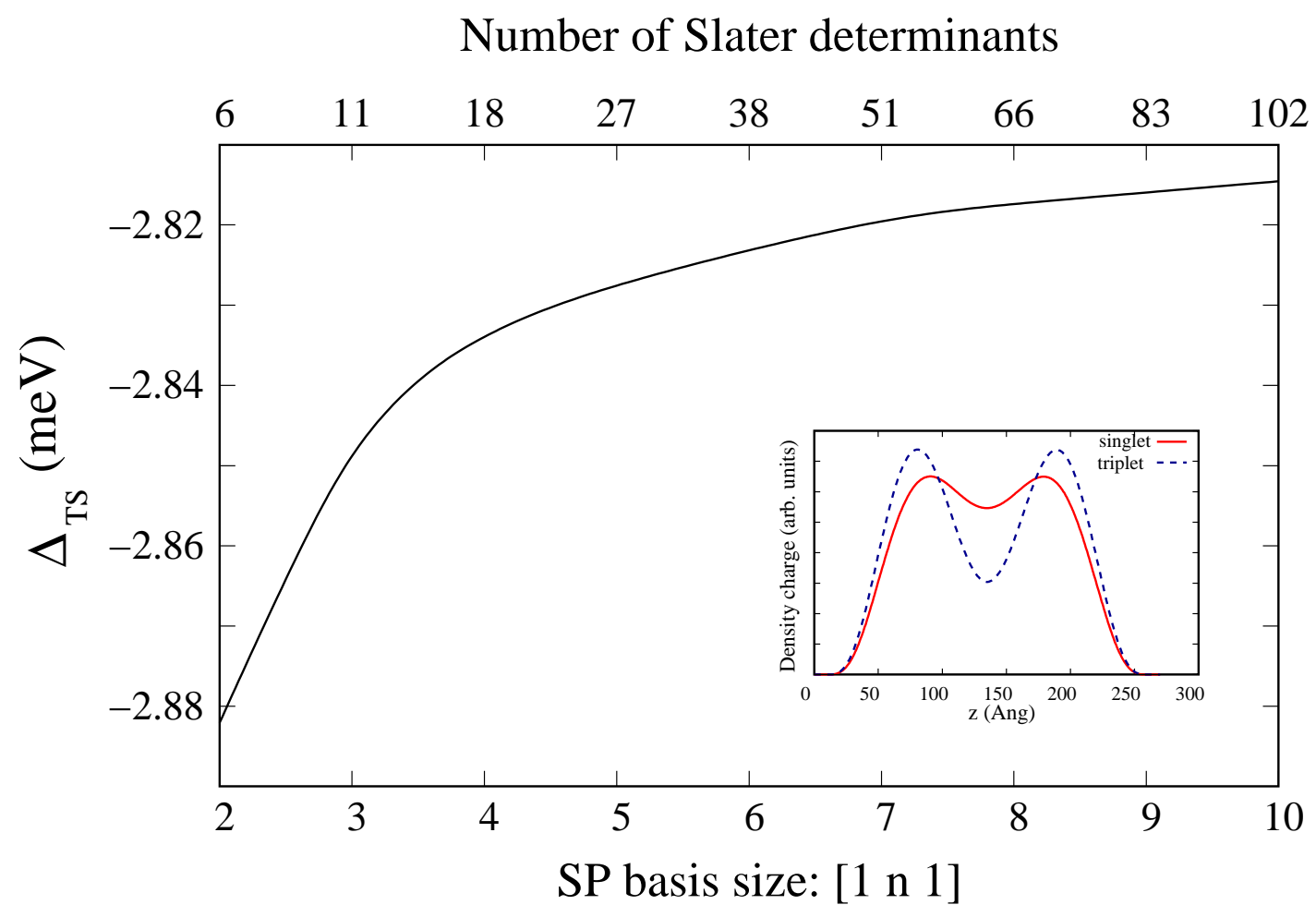

Figure 2. FCI singlet-triplet energy gap $\Delta_{T S}$, corresponding to a CdSe $R=20 \AA$, $L=200 \AA \mathrm{NR}$, calculated with a SP $1-n-1$ basis set vs. $n$. Inset: $R=0$ profile of the FCI singlet (solid line) and triplet (dashed line) charge densities along the NR $z$ axis. 
Correlation in narrow nanorods: a variational potential-configuration interaction scheme10

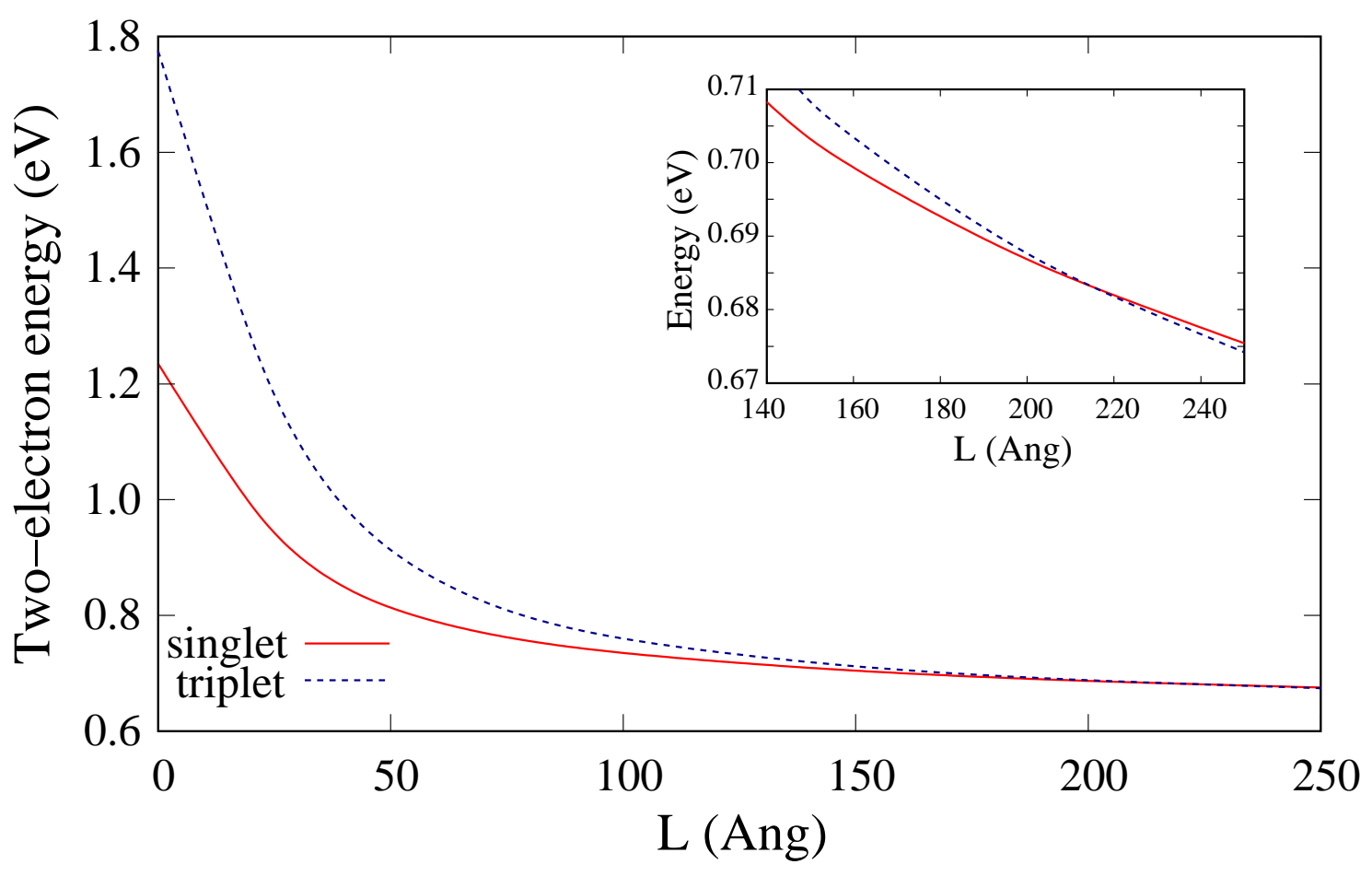

Figure 3. FCI calculations, employing a 1-6-1 HF basis set, of the singlet (solid line) and triplet (dashed line) states of a $R=20 \AA$ two-electron semiconductor CdSe NR vs. the length $L$. Inset: close-up of the large length region.

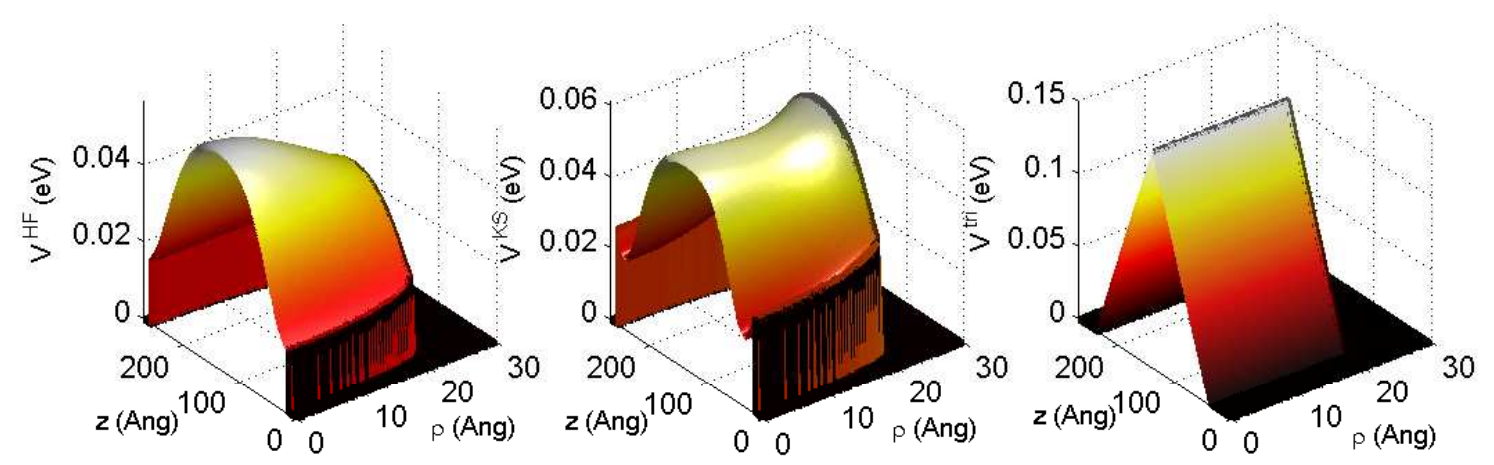

Figure 4. Mean field HF (left), KS (center) and, variationally optimized $v^{\text {tri }}$ (right) potentials of a $R=20 \AA, L=200 \AA$ two-electron CdSe NR. 


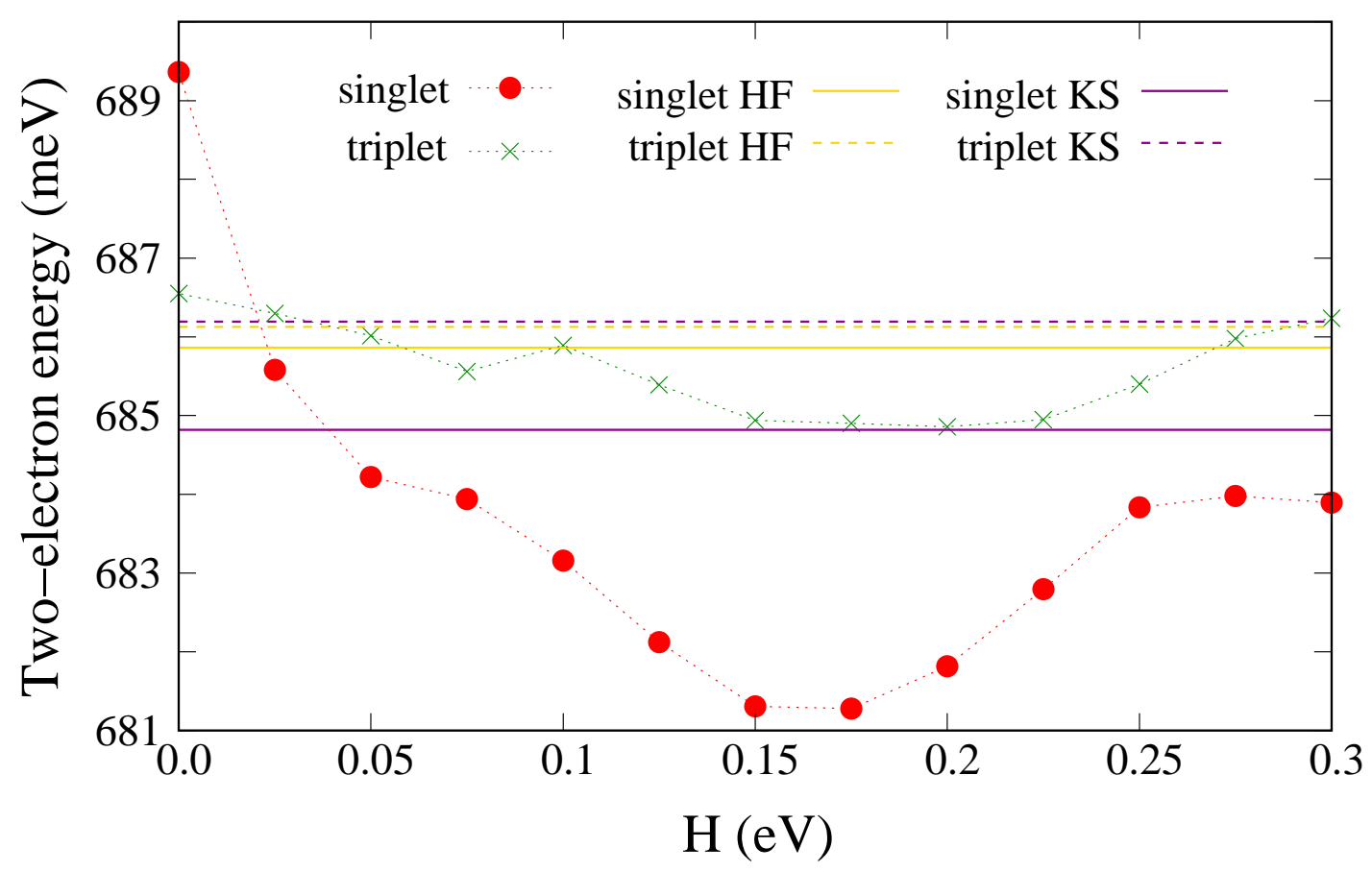

Figure 5. FCI optimization of the variational triangle-like $v^{\text {tri }}(H)$ potential vs. its height $H$, in the case of a $R=20 \AA, L=200 \AA$ CdSe NR, employing a 1-10-1 basis set. Singlet (dots) and triplet (crosses) energies are represented. Horizontal solid (singlet) and dashed (triplet) lines representing HF-FCI (dark lines) and KS-FCI (light lines) are also enclosed. 
Correlation in narrow nanorods: a variational potential-configuration interaction scheme12

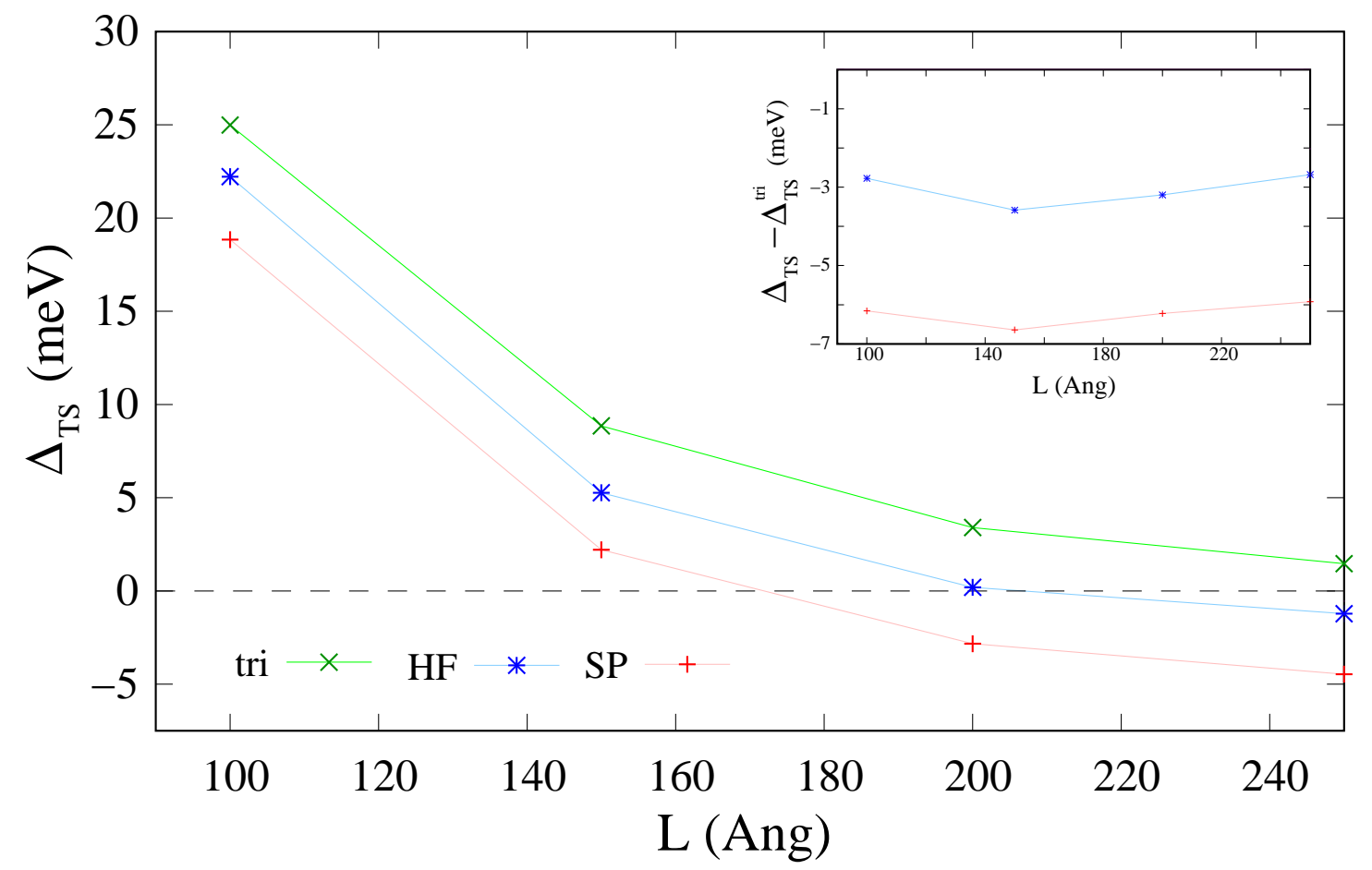

Figure 6. FCI singlet-triplet gap $\Delta_{T S}$ obtained with SP, $v^{H F}$ and $v^{t r i}$ vs. $L$ of a $R=20 \AA$ two-electron semiconductor CdSe NR. In the inset, differences between the calculated SP and $v^{H F}$ and the $v^{t r i}$ singlet-triplet gaps. 